\title{
PERCEPCIONES DE UN ESTUDIANTE DE DOCTORADO DE OTRO PAIS SOBRE LOS PROCESOS DE EVALUACIÓN FORMATIVA Y COMPARTIDA EN FORMACIÓN INICIAL DEL PROFESORADO DE ESPAÑA
}

\author{
Perceptions of a $\mathrm{PhD}$ student from another country about formative and shared \\ assessment processes in teacher education training of Spain
}

Perceções de um estudante de doutoramento de outro país sobre os processos de avaliação formativa e partilhada na formação inicial de professores de Espanha

\author{
André Pisco de Moura (1)
}

Víctor M. López-Pastor (2)

Roberto Monjas Aguado (3)

Financial support: The work of the first author is supported by Fundação para a Ciência e Tecnologia (FCT). PhD grant: SFRH/BD/137848/2018

(1) Universidad de Porto, Facultad de Deporte, Portugal. Teléfono: +351 917522046. Correo electrónico: luisandrepmoura@hotmail.com

(2) Universidad de Valladolid, España. Teléfono: +34 921112321. Correo electrónico: vlopez@mpc.uva.es

(3) Universidad de Valladolid, España. Teléfono: +34 921112321. Correo electrónico: rmonjas@mpc.uva.es

\section{Resumen}

En el presente trabajo se realiza un análisis de los procesos de evaluación formativa y compartida empleados en la Facultad de Educación de Segovia desde la óptica de un investigador portugués. Los datos fueron recogidos mediante diferentes técnicas cualitativas: observación participante, diálogos con el profesorado y análisis de documentos. El investigador observó 30 clases de dos asignaturas del Grado de Maestro en Educación Infantil (una: 35 alumnas y otra: 30 alumnas y 15 alumnos) y 15 clases en Educación Primaria (10 alumnas y 18 alumnos). Los resultados son presentados en forma de diálogo entre el investigador y los profesores sobre los temas: (1) evaluación entre iguales y (2) procesos de evaluación formativa y compartida y aprendizaje dialógico llevados a cabo. Se identificaron algunas dificultades en los procesos de evaluación entre estudiantes y se comprobó que las asignaturas están centradas en el alumnado, con procesos constantes de feedback, evaluación y dialogo.

Palabras clave: Investigador externo; evaluación formativa; evaluación compartida; formación del profesorado

Percepciones de un estudiante de doctorado de otro país sobre los procesos de evaluación formativa y compartida en formación inicial del profesorado de España 


\begin{abstract}
This work presents an analysis of formative and shared assessment processes used in Faculty of Education of Segovia by a Portuguese researcher. Data were collected through different qualitative techniques: participant observation, dialogues with teachers and document analysis. This researcher observed 30 classes of two subjects of bachelor's in teaching in Kindergarten Education (one: 35 girls and another: 30 girls and 15 boys) and 15 classes of Primary Education (10 girls and 18 boys). Results were presented through dialogues among the research and teachers, considering the following themes: (1) peer assessment and (2) formative and shared assessment processes and dialogic learning developed during these subjects. Some difficulties were identified on peer assessment processes and was ascertained that these subjects are student centered and occur through continuous processes of feedback, assessment and dialogue.
\end{abstract}

Keywords: External researcher; formative assessment; shared assessment; teacher education

\title{
Resumo
}

No presente trabalho realiza-se uma análise dos processos de avaliação formativa e partilhada utilizados na Faculdade de Educação de Segóvia, partindo da perspetiva de um investigador português. Os dados foram recolhidos através de diferentes técnicas qualitativas: observação participante, diálogos com os professores e análise de documentos. O investigador observou 30 aulas de duas disciplinas da Licenciatura em Ensino na Educação Pré-Escolar (uma: 35 alunas e outra: 30 alunas e 15 alunos) e 15 aulas na Educação Primária (10 alunas e 18 alunos). Os resultados são apresentados em forma de diálogo entre o investigador e os professores, tendo em conta, os seguintes temas: (1) avaliação entre iguais e (2) processos de avaliação formativa e partilhada e aprendizagem dialógica levados a cabo. Identificam-se algumas dificuldades nos processos de avaliação entre alunos e comprovou-se que estas disciplinas colocam o aluno no centro do processo, através de processos constantes de feedback, avaliação e diálogo.

Palavras-chave: Investigador externo; avaliação formativa; avaliação partilhada; formação de professores

Percepciones de un estudiante de doctorado de otro país sobre los procesos de evaluación formativa y compartida en formación inicial del profesorado de España 


\section{Introducción}

España tiene cierta tradición en el desarrollo de procesos de evaluación formativa y compartida (EFyC) en educación superior (López-Pastor, 2009; López-Pastor \& PérezPueyo, 2017). Un estudiante de doctorado portugués, interesado en esta temática de cara a su tesis doctoral, decidió realizar una estancia de investigación para conocer y analizar los procesos de EFyC en la Facultad de Educación de Segovia.

\section{Método}

Este trabajo analiza las percepciones del investigador externo sobre los procesos de EFyC desarrollados. Se observaron 30 clases de dos asignaturas del Grado de Maestro en Educación Infantil (una: 35 alumnas y otra: 30 alumnas y 15 alumnos) y 15 clases en Educación Primaria (10 alumnas y 18 alumnos) de dos profesores durante dos meses (abril-mayo 2018/19). Se utilizan categorías inductivas en base a las notas de campo y se recogen datos a través de observación participante, diálogos individuales con los profesores y análisis de documentos (material utilizado en las asignaturas). Según Becker y Geer (1970) el mejor método para comprender totalmente los datos es la participación directa y observación de lo fenómeno que estamos estudiando. Los diálogos permiten clarificar dudas y razones de los profesores para trabajar así.

\section{Resultados}

Los presentamos como un diálogo entre investigador y profesores sobre dos categorías de análisis: (1) evaluación entre iguales y (2) procesos de EFyC y aprendizaje dialógico utilizados.

\section{1-Evaluación entre iguales}

A menudo el alumnado tiene que realizar procesos de evaluación entre iguales. Por ejemplo, al finalizar las sesiones prácticas que han impartido sus compañeros/as se les pide que realicen un análisis didáctico y de las competencias docentes observadas. El diálogo se centra en algunas de las dificultades observadas por el investigador.

Investigador (I)-Veo que los estudiantes tienen dificultad en realizar valoraciones de sus compañeros al finalizar cada sesión práctica, aunque tengan un gran estímulo por parte del profesorado. Las relaciones personales/afectivas parecen afectar esa valoración.

Profesor 1 (P1)-Si, es cierto. Creo que influyen dos cosas: la falta de hábito y las implicaciones afectivas. Necesitan realizar análisis de varias sesiones hasta que aprenden a hacerlo. El aspecto emocional es que la tendencia natural del alumnado es guiarse más por la relación afectivo-social 
con las personas a las que evalúa que por un razonamiento técnico y profesional. Es otro aprendizaje que tienen que realizar a lo largo de la asignatura y de la carrera; entender que no es un ataque o una crítica personal, sino una reflexión sobre un ejemplo de intervención docente.

Profesor 2 (P2)-Es importante que entiendan la importancia de hacer valoraciones positivas que favorezcan el acercamiento en el proceso evaluador, de manera que desarrollen un sentido crítico y constructivo, que permita que el proceso evaluador sirva para el aprendizaje y la mejora profesional.

El investigador también comenta sobre las valoraciones cuantitativas y cualitativas que el alumnado realiza sobre las competencias docentes observadas:

I-He observado que los alumnos fácilmente asignan un valor a sus colegas en los procesos de análisis y reflexión colectiva, pero tienen dificultad en justificarlo.

P1-Queremos que aprendan a evaluar utilizando diferentes procesos: con una escala numérica y también justificándolo de forma verbal. En todo caso, se les deja muy claro que no son notas, que son simples procesos de valoración de las competencias que han observado, con la finalidad de que aprendan a dominar dichas competencias docentes cuando tengan que ser maestros. En ningún caso sus valoraciones van a influir en la evaluación que los profesores realizan de sus compañeros.

\section{2-Procesos de evaluación formativa, compartida y de aprendizaje dialógico} utilizados

El investigador cree que este tipo de sistemas de evaluación es beneficioso para la formación de futuros maestros, pero considera necesario que las sesiones prácticas se realicen también con niños/as:

I-Creo que con estos sistemas de evaluación los estudiantes adquieren una mejor formación en competencias docentes que si no hubieran pasado por estas vivencias, pero era importante hacer también sesiones prácticas con niños, para que sea una práctica más real que la simple simulación con sus compañeros.

P1-Completamente de acuerdo con la primera afirmación. Nosotros también lo vemos así, por eso las utilizamos. Respecto a lo de hacerlo con niños reales, en vez de solo hacer la simulación práctica con sus compañeros, en esta asignatura es una opción voluntaria, como trabajo y puntos extra.

El investigador también se interesa por las valoraciones que realizan los egresados tras finalizar la carrera y acceder a un puesto de trabajo, fundamentalmente sobre este tipo de sistemas de evaluación y aprendizaje.

I-¿Cuál es el feedback que tenéis de antiguos estudiantes que están como maestros/as en la escuela? ¿Cómo se sienten empleando los procesos de evaluación?

P1-El feedback es positivo. Vivimos en una ciudad pequeña y es habitual encontrarse a los estudiantes por la calle después de haberse graduado. La mayoría de ellos hacen valoraciones positivas sobre el aprendizaje profesional desarrollado. Suelen centrarse más en el proceso de 
aprendizaje que en la evaluación. En los últimos cinco años estamos realizando algunos estudios preguntando a antiguos egresados por sus percepciones sobre los sistemas de evaluación vividos en su carrera. En la mayoría de los casos las valoraciones son positivas y reconocen que les ha ayudado a generar sistemas de evaluación formativa y compartida en los centros en los que trabajan.

P2-La idea es contribuir a que ellos mismos decidan qué sistema de evaluación van a desarrollar en su práctica docente y consideran que les ha ayudado a dar sentido a su planteamiento como docentes.

\section{Conclusiones}

Los resultados muestran un primer mapa de las percepciones del investigador sobre los procesos de EFyC observados. El alumnado tiene dificultad con los procesos de evaluación entre iguales. Estas asignaturas están centradas en el alumnado, utilizan procesos constantes de feedback, evaluación y diálogo entre profesorado y alumnado que estimulan su participación.

La formación docente es un proceso que incluye el aprendizaje sobre la evaluación y para ello es necesario que el alumnado en formación conozca y ponga en práctica diferentes sistemas de evaluación, siendo conscientes al mismo tiempo de sus vivencias previas para reflexionar qué tipo de evaluación es el más coherente con su forma de entender la docencia.

En definitiva, haber vivido estos procesos de aprendizaje y evaluación en formación inicial del profesorado prepara mejor al alumnado para enseñar y evaluar en el futuro.

\section{Referencias}

Becker, H., \& Geer, B., (1970). Participant Observation and Interviewing: A Comparison. In W. J. Filstead (Eds.), Qualitative Methodology. (pp. 133-141). Chicago: Markham

López-Pastor, V. M. (Coord.) (2009). Evaluación Formativa y Compartida en Educación Superior. Narcea: Madrid.

López-Pastor, V. M., \& Pérez-Pueyo, A. (Coords.) (2017). Evaluación formativa y compartida en educación: experiencias de éxito en todas las etapas educativas. León: Universidad de León. (e-book). Recuperado de https://buleria.unileon.es/handle/10612/5999 